\title{
In Situ Plant Canopy and Potting Medium Temperatures under Two Greenhouse Temperature Regimes
}

\author{
Richard J. McAvoy \\ Plant Science Department, University of Connecticut, Storrs, CT 06269
}

Additional index words. Euphorbia pulchetima, day/night temperature

Abstract. Root-zone and plant canopy temperatures were continuously monitored as a poinsettia (Euphorbia pulcherrima Willd. ex JSI.) crop was grown in the greenhouse under warm day/cool night $[(+)$ DT-NT] or cool day/warm night [( - ) DT-NT] temperature regimes. Day temperatures were imposed from 0900 to $1700 \mathrm{HR}$. Light levels photosynthetic photon flux (PPF) and outside ambient air temperatures were also monitored. Temperature differences between the root-zone and plant canopy microenvironments were most extreme during the night-to-day and day-to-night temperature transition periods. The temperature difference between the plant canopy and the root zone following temperature transition periods had been previously identified as a critical factor affecting stem elongation. Overall poinsettia height was consistently shorter under the (-) DT-NT than under the (+) DT-NT environment.

Low air temperature pulses stimulate the phasing or timing of the daily cycle of stem elongation and the stem extension rate (Lecharny et al., 1985). The change in air temperature rather than the absolute air temperature was identified as a critical factor affecting stem elongation in Chenopodium rubrum L. Recently, the absolute air temperature difference between the day and night periods was identified as an environmental factor that affects stem elongation and axillary shoot development of certain floricultural crops (Erwin et al., 1989a). Moe and Heins (1990) reported that plants produced shorter internodes and increased axillary branching when night air temperatures were warmer than day air temperatures. Under cool night/warm day air temperatures, increased internode length and decreased axillary branching was observed.

Plant height and axillary shoot development are also affected by root-zone heating. Brown and Ormrod (1980) reported decreased plant height and increased axillary shoot initiation in roses produced with bottom heat. Janes and McAvoy (1982) reported that poinsettias produced with bottom teat were shorter with more prominent axillary shoot development than when bottom teat was not provided. Merritt and Kohl 1982) reported increased axillary shoot development of petunia with root zone heating under short day conditions. However, under long days they observed decreased branching and increased stem elongation when the root one was heated. Wulster and Janes (1984)

Received for publication 5 Aug. 1991. Accepted for publication 18 Mar. 1992. Scientific Contribution no. 1349 of the Storrs Agricultural Experrent Station, Univ. of Connecticut. Appreciation expressed to Eake Poinsettias for donating plant material for this study. The cost of publishing this paper was defrayed in part by the payment of page charges. Under postal regulations, this paper therefore must be hereby marked advertisement solely to indicate this fact. reported an increased number of axillary shoots developed on unpinched poinsettias when root-zone heating was applied 24 $\mathrm{h} \cdot$ day $^{-1}$. However, when root-zone heat was applied only during the night (1600 to 0800 $\mathrm{HR}$ ) to pinched poinsettias, axillary shoot count was unaffected.

With $C$ rubrum, the stem elongation rate increased when soil temperature was lower than the temperature in the aerial portion of the plant (Lechamy et al., 1985). In their study, low air temperature pulses were most effective when applied at the end of a dark period.

This study was conducted to quantify in situ temperature changes in the canopy and potting medium (root zone) microenvironments under two day/night air temperature regimes, and to determine the response of two poinsettia cultivars to these environments.

Rooted cuttings of E. pulcherrima cvs. Eckespoint Lilo (Lila) and Annette Hegg Diva

Table 1. Influence of day/night temperature environment and cultivar on final poinsettia plant characteristics.

\begin{tabular}{|c|c|c|c|c|}
\hline \multirow[b]{2}{*}{ Variable } & \multicolumn{2}{|c|}{ Plant } & \multicolumn{2}{|c|}{ Axillary shoots } \\
\hline & $\mathrm{Ht}(\mathrm{cm})^{2}$ & $\begin{array}{c}\text { Total dry } \\
\text { wt }(g)\end{array}$ & No. & Length $(\mathrm{cm})$ \\
\hline \multicolumn{5}{|c|}{ Temperature regime (TR) } \\
\hline $\begin{array}{l}\text { (+) DT-NT } \\
(-) \text { DT-NT }\end{array}$ & $\begin{array}{l}32.7 \\
29.3^{*}\end{array}$ & $\begin{array}{l}17.7 \\
18.2\end{array}$ & $\begin{array}{l}4.7 \\
5.8\end{array}$ & $\begin{array}{l}6.2 \\
8.8\end{array}$ \\
\hline \multicolumn{5}{|c|}{ Cultivar $(C)$} \\
\hline $\begin{array}{l}\text { Diva } \\
\text { Lilo }\end{array}$ & $\begin{array}{l}30.2 \\
31.8\end{array}$ & $\begin{array}{l}17.7 \\
18.2\end{array}$ & $\begin{array}{l}6.9 \\
3.6^{*}\end{array}$ & $\begin{array}{l}8.4 \\
6.6\end{array}$ \\
\hline \multicolumn{5}{|c|}{$T R \times C$} \\
\hline $\begin{array}{l}(+) \text { DT-NT } \times \text { Diva } \\
(-) \text { DT-NT } \times \text { Diva } \\
(+) \text { DT-NT } \times \text { Lilo } \\
(-) \text { DT-NT } \times \text { Lilo }\end{array}$ & $\begin{array}{l}31.3 \\
29.1 \\
34.1 \\
29.5\end{array}$ & $\begin{array}{l}18.8 \\
16.5 \\
16.5 \\
19.9\end{array}$ & $\begin{array}{l}7.2 \\
6.6 \\
2.2 \\
5.0\end{array}$ & $\begin{array}{l}9.0 \\
7.8 \\
3.4 \\
9.7^{* *}\end{array}$ \\
\hline
\end{tabular}

${ }^{2}$ Main effect values represent means of 10 observations. Values for interactions represent means of five observations.

$* * * *$ Significant at $P \leq 0.05$ or 0.01 , respectively, according to the ANOVA F test. All other treatment effects nonsignificant at $\mathrm{P} \leq 0.05$.
Starlight (Diva) were planted on 30 Aug 1989 into 1.6.liter pots containing a 1 peat : 1 perlite : 1 vermiculite medium (by volume) amended with (all in $\mathrm{kg} \cdot \mathrm{m}^{-3}$ ) 6.0 dolomitic limestone, 1.6 superphosphate, 0.6 $\mathrm{Ca}\left(\mathrm{NO}_{3}\right)_{2}$, and 0.095 fritted trace elements. Plants were fertilized with $16.5 \mathrm{~mm} \mathrm{~N}$ at every irrigation beginning on 30 Aug. by alternately applying $19 \mathrm{~N}-2.2 \mathrm{P}-21.3 \mathrm{~K}$ and 15N-7P-15.1K. Photoperiod lighting (2200 to $0200 \mathrm{HR}$ ) was provided from 7 Sept. through 5 Oct.

The poinsettias were divided into two groups on 19 Sept. and each group was moved to one section of a partitioned greenhouse. The day temperature was imposed from 0900 to 1700 HR daily. Day temperature (DT) and night temperature (NT) settings were 21.0 and $15.5 \mathrm{C}( \pm 0.5 \mathrm{C})$ in one section and 15.5 and $21.0 \mathrm{C}$ in the other, resulting in positive $(+)$ DT-NT and negative ( - ) DT-NT environments, respectively. Plants were not pinched.

Within the two greenhouse environments, temperature and light levels were continuously monitored at 5-sec intervals and hourly averages recorded using a Campbell Scientific 21X micrologger (Logan, Utah). Thermocouples were located in the plant canopy (midcanopy) and in the potting medium (midpot) at four locations within each temperature regime. In addition, the ambient temperature outside the greenhouse and photosynthetically active radiation (PAR) at canopy level were monitored.

The two cultivars were set out using a randomized block design with five replications in each of the two DT-NT regimes. Analysis of variance (ANOVA) was conducted for each temperature regime, and, in addition, an ANOVA over sites was conducted for the combined data (Gomez and Gomez, 1984).

Poinsettias were harvested on 19 Dec. and plant height, number of axillary shoots, length of each axillary shoot, and dry weight of leaves, bracts, and stems were determined.

During the first hour of DT treatment (0900 to $1000 \mathrm{HR}$ ), canopy temperature rapidly 


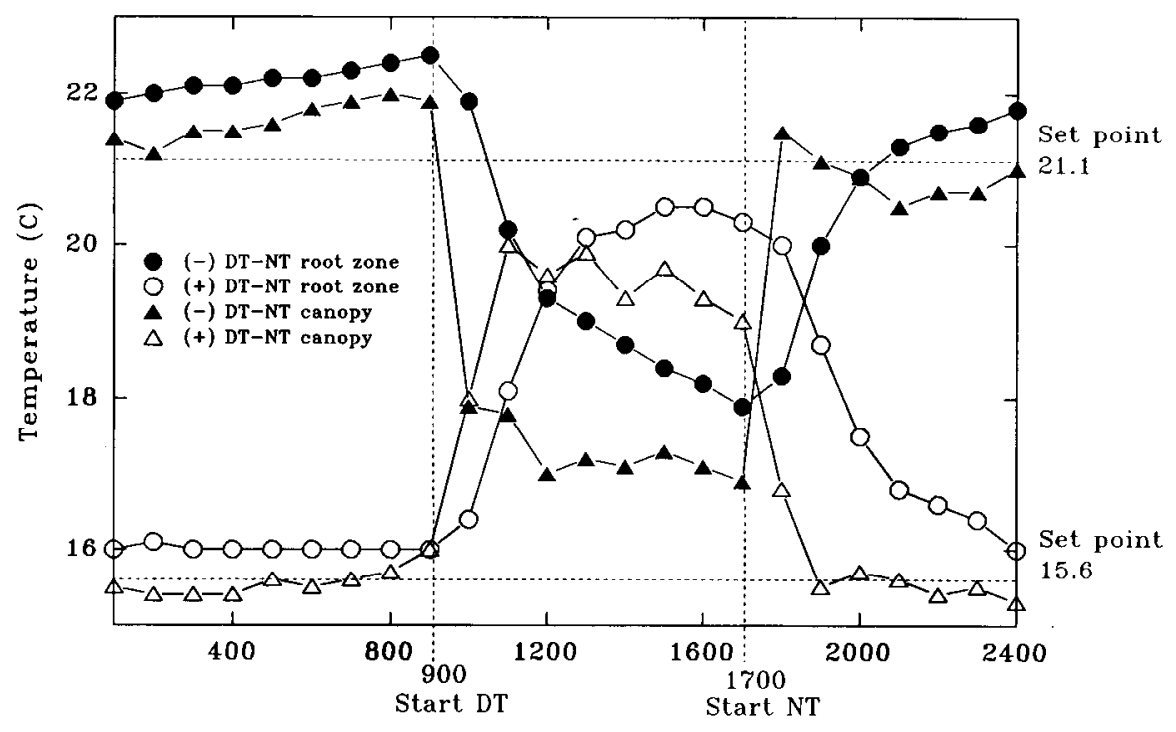

Time (hours)

Fig. 1. Average diurnal temperature in the plant canopy and the root zone microenvironments under two day/night temperature regimes. Day temperature (DT) began at $0900 \mathrm{HR}$ and night temperature (NT) at $1700 \mathrm{HR}$ (see vertical dashed lines). Day/night temperatures set points were either 21.0 or 15.5C (depending on the DT-NT regime employed, see horizontal dashed lines). Data points represent hourly means from 15 to 25 Nov. 1989.

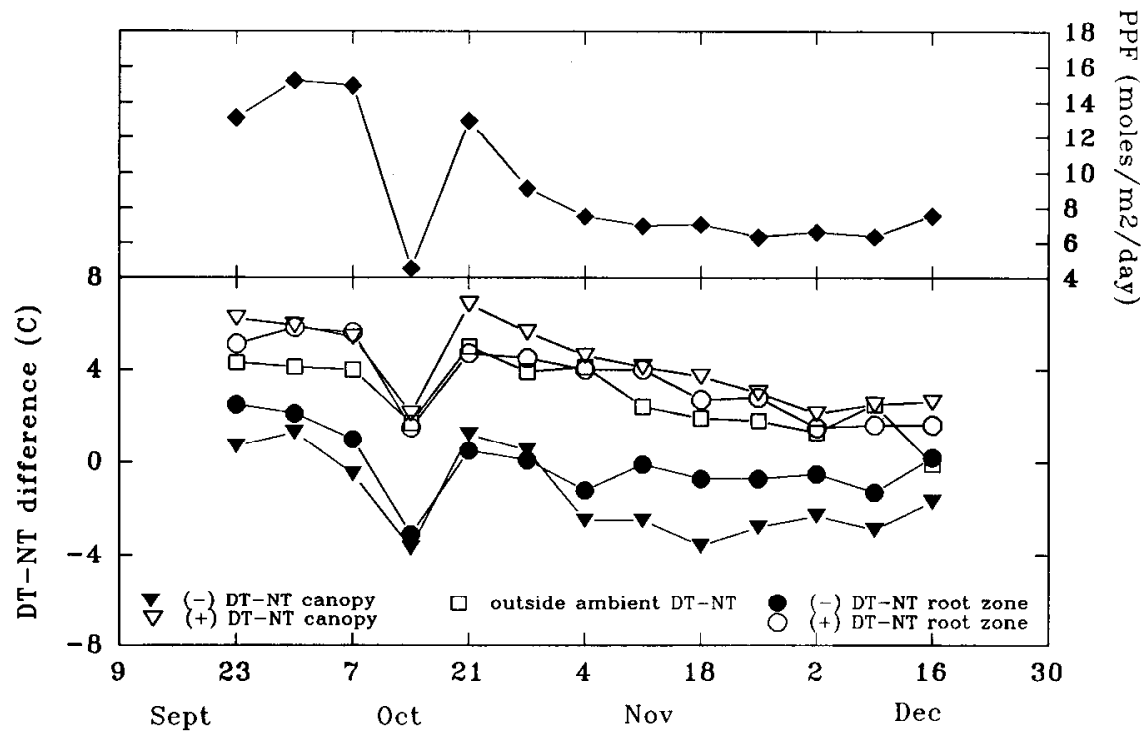

Time

Fig. 2. Average day - night temperature difference (DT-NT) averaged over weekly intervals as measured in the canopy and root zone of plants in the positive $(+)$ and negative $(-)$ day - night air temperature environments and the air outside the greenhouse (bottom). Average weekly photosynthetic photon flux (PPF) in $\mathrm{mol} \cdot \mathrm{m}^{-2} \cdot \mathrm{day}^{-1}$ is also presented (top).

equilibrated to the new set point temperature (Fig 1). However, in both the (+) and (-) DT-NT environments, potting medium (root zone) temperatures remained closer to NT than DT during the first hour of the DT treatments (Fig 1). The largest difference between root zone and canopy temperatures occurred during the DT/NT and NT/DT transition periods. Under the (-) DT-NT environment, the root zone was warmer than the canopy during the first hour of the NT/DT transition period. Under the (+) DT-NT environment, the root zone was cooler than the similar relationship with ambient environmental conditions (Fig 2).

Poinsettias in the (+) DT-NT environment were significantly taller $(11.6 \%)$ than plants in the (-) DT-NT environment (Table 1), a response consistent with previous reports for poinsettias grown under $(+)$ and (-) DT-NT conditions (Erwin et al., 1989b). Under the ( + ) DT-NT environment, the average overall heights of 'Diva' and 'Lilo' were greater $(7.2 \%$ and $15.6 \%$, respectively) than under the (-) DT-NT environment. On average, 'Lilo' and 'Diva' attained statistically similar final heights and total dry weights (Table 1).

When poinsettias were grown in a $(+)$ DT-NT regime, cooler root zones and warmer canopies existed during the hours immediately following the NT/DT transition period (Fig. 1). The temperature difference between the root zone and the plant canopy following the NT/DT transition period has been identified as the factor that modulates the stem elongation response to environmental stimuli (Lechamy et al., 1985). In their study, the stem elongation rate of $C$. rubrum was promoted by a lower root zone than canopy temperature following the NT/DT transition period under a $(+)$ DT-NT regime. In comparison, the stem elongation rate was reduced when root-zone temperature was regulated to follow air temperature under the same (+) DT-NT regime.

A significant day/night air temperature by cultivar interaction for axillary shoot length was observed with poinsettia (Table 1). 'Diva' produced axillary shoots that averaged $15.4 \%$ longer under the (+) DT-NT environment than under the (-) DT-NT environment. Whereas, with 'Lilo', axillary shoots under the (+) DT-NT environment averaged 2.8 times shorter than those in the (-) DT-NT environment.

'Lilo' did not branch as freely as 'Diva'. 'Diva' produced significantly more lateral shoots (6.9) than 'Lilo' (3.6). Under the (+) DT-NT environment, 'Lilo' averaged only 2.2 axillary shoots per plant as compared to the 5.0 axillary shoots 'Lilo' produced under the (-) DT-NT environment. In addition, 'Lilo' initiated axillary shoots late in the growth cycle and toward the top of the stem in the $(+)$ DT-NT environment. In the $(-)$ DT-NT environment, 'Lilo' initiated axillary shoots early in the growth cycle and from nodes lower on the stem. As a result, in the (-) DT-NT greenhouse, 'Lilo' produced axillary shoots that developed over a longer period before bracts developed than those in the (+) DT-NT environment. In contrast, 'Diva' initiated more axillary shoots than 'Lilo' and in roughly equal numbers under the two temperature environments, and the average length tended to be shorter in the (-) DT-NT environment.

The greenhouse environment can vary significantly over relatively short periods of time as environmental conditions such as PPF and outside ambient air temperatures fluctuate. Therefore, the timing of axillary shoot initiation and internode development influences the growth response. 
The diurnal temperature profile observed in this study (Fig. 1) indicates that a rapid change in air temperature increased the temperature difference between the root zone and canopy of poinsettia, one of the factors affecting stem elongation (Lechamy et al., 1985). Two environmental factors regulate stem elongation. The first, a change in air temperature such as a cool NT, cool DT, or a cool-temperature pulse, triggers the phasing or timing of the stem elongation response. It is the change in air temperature that initiates the daily stem elongation cycle and thus controls when the plant is most responsive to environmental stimuli that affect stem elongation. When $C$. rubrum was grown under a ( + ) DT-NT regime, maximum stem elongation occurred during the ensuing day period and stem elongation rates were high. When this species was grown under a (-) DT-NT regime, cool day temperatures triggered the stem elongation response to occur during the dark period and stem elongation rates were lower.

The second environmental factor regulating stem elongation is the canopy-root-zone temperature difference. This temperature difference affects the magnitude of the stem elongation response to environmental stimuli but not the timing of the elongation response (Lechamy et al., 1985). When canopy-rootzone temperature differences increase (i.e., are positive), stem elongation rates increase. Root zone heating during the day reduces the canopy-root-zone temperature difference, reducing the magnitude of the stem elongation response. Thus, (-) DT-NT regimes (Erwin et al., 1989a; Moe and Heins, 1990), and continuous $(24 \mathrm{~h})$ root zone heating under (+) DT-NT regimes (Janes and McAvoy, 1983; Wulster and Janes, 1984), may produce similar plant growth responses by regulating separate mechanisms controlling the stem elongation response.

In situ, the influence of the canopy-rootzone temperature difference on the stem elongation rate would depend on the magnitude of the temperature difference, whether the difference was positive or negative (i.e., canopy temperature was higher or lower than root-zone temperature), and the duration over which the temperature differences persists following the onset of the stem elongation period. DT-NT regimes, container volumes, or other physical conditions that influence the temperature dynamics in the plant canopy and the root zone during the inductive stem elongation cycle, could presumably affect the stem elongation response. If these conditions favored root zone temperatures higher or equal to canopy temperatures during the daily stem elongation cycle, lower stem elongation rates would be expected. Finally, the plant response to DT-NT regimes varies between species (Erwin, 1991) and, as demonstrated in this study, between cultivars (i.e., axillary shoot development of 'Lilo' vs. 'Diva'). Therefore, the effects of DT-NT regimes and canopy-root-zone temperature differences reported for $C$. rubrum may differ both quantitatively and qualitatively for E. pulcherrima.

\section{Literature Cited}

Brown, W.W. and D.P. Ormrod. 1980. Soil temperature effects on greenhouse roses in relation to air temperature and nutrition. J. Amer. Soc. Hort. Sci. 105(1):57-59.

Erwin, J.E. 1991. .Temperature effects on plant month. Proc. of the 12th Annu. Conf. on Hydroponics. St Charles, Ill, p 1-6.

Erwin, J.E., R.D. Heins, and M.G. Karlsson. 1989a. Thermomorphogenesis in Lilium longiflorum. Amer. J. Bot. 76(1):47-52.

Ekin, J.E., R. Heins, R. Birghage, and W. Carlson. 1989b. How can temperature be used to control plant stem elongation. Minnesota State Florists Bul. 38(3):1-4.

Gomez, K.A. and A.A. Gomez. 1984. Statistical procedures for agricultural research. 2nd ed. Wiley, New York.

Janes, H.W. and R. McAvoy. 1982. Effect of root zone heating on growth of poinsettia. J. Amer. Soc. Hort. sci. 107(3):525-530.

Janes, H.W. and R. McAvoy. 1983. Deleterious effects of cool air temperatures reversed by rootzone warming of poinsettia. HortScience 18:363364.

Lechamy, A., M. Schwall, and E. Wagner. 1985. Stem extension rate in light-grown plants. Plant Physiol. 79:625-629.

Merritt, R.H. and H.C. Kohl, Jr. 1982. Effect of root temperature and photoperiod on growth and crop productivity efficiency of petunia. J. Amer. Soc.. Hort. Sci. 107:997-1000.

Moe, R. and R. Heins. 1990. Control of plant morphogenesis and flowering by light quality and temperature. Acta Hort. 272:81-89.

Wulster, G.J. and H.W. Janes. 1984. The effect of elevated root zone temperatures of various duration on growth and development of several poinsettia cultivars. Acta Hort. 148:835-842. 